\title{
Crossovers in spin-boson and central spin models
}

\author{
P. C. E. Stamp ${ }^{1}$, I. S. Tupitsyn ${ }^{2}$ \\ 1 Canadian Institute for Advanced Research, \\ and Physics Dept., University of British Columbia, \\ 6224 Agricultural Rd., Vancouver, \\ B.C., Canada V6T $1 Z 1$ \\ 2 Russian Science Centre "Kurchatov Institute", \\ Moscow 123182, Russia
}

\begin{abstract}
We discuss how the crossovers in models like spin-boson model are changed by adding the coupling of the central spin to localised modes- the latter modelled as a 'spin bath'. These modes contain most of the environmental entropy and energy at low $T$ in solid-state systems. We find that the low $T$ crossover between oscillator bath and spin bath dominated decoherence, occurring as one reduces the energy scale of the central spin, is characterised by very low decoherence- we show how this works out in practise in magnetic insulators. We then reconsider the standard quantum-classical crossover in the dynamics of a tunneling system, including both spin and oscillator baths. It is found that the general effect of the spin bath is to broaden the crossover in temperature between the quantum and classical activated regimes. The example of tunneling nanomagnets is used to illustrate this.
\end{abstract}

PACS numbers:

\section{1: INTRODUCTION}

In the book of Weiss on quantum dissipative phenomena 1] one finds a very nice summary of results on the crossover between quantum tunneling and classical activation for a single tunneling coordinate coupled to a bath of oscillators (see Chapters 10-17, particularly 14 and 16) . This kind of problem has a long and interesting history, beginning with work of Kramers [2] in 1940. The oscillator bath models assume that each bath mode is weakly perturbed, and then the description of the bath by oscillators is well known to be correct. Many physical systems are very accurately described by such models [1, 3, 4, 6] , and they are central to much of reaction rate chemistry as well. Typically one studies either a particle tunneling from a trapped state to an open continuum of states (the dissipative tunneling problem), or a doublewell system in which a particle has to go from one well to another (the dissipative 2-well problem). One has a range of temperatures in which both activation and tunneling processes are important. Both the width of the crossover regime and the detailed dependence of transition rates, as a function of temperature and applied bias, are of interest [1, 5]. In the 2 well problem, the 'quantum limit', where only the 2 lowest levels of the 2-well system are relevant (assuming a weak bias between the wells), has been studied very extensively. This is the 'spin-boson model', in which a 2-level system couples to the oscillator environment.

Another interesting application of the spin-boson model is to the problem of qubits in quantum information processing (QUIP). The central issue here is the study of decoherence in the dynamics of the qubit, and how it depends on both simple things like applied fields, temperature, etc., and in a more complex way on the de- tailed nature of the bath, and its coupling to the qubit. It turns out that at the low temperatures that are appropriate for QUIP, or for any other large scale quantum coherence, the oscillator bath models are no longer adequate to describe all the physics. In many systems the decoherence is controlled largely by the coupling to localised modes, such as defects, tunneling charges, paramagnetic spins, or nuclear spins, and this environment of localised modes cannot in general be modelled by oscillators- it can however be described as a set of spins 7] (the 'spin bath'). There is now extensive experimental evidence for the key role of such modes in experiments on Cooper pair box qubits [8], SQUID qubits [9, 10], and in molecular magnets 11, 12, but the importance of these modes is already rather obvious just from an estimation of their coupling to these systems. There have been a fair number of theoretical studies of spin bath environments. Early partial studies, in various contexts, include refs. [13, 14, 15, 16]; later work has concentrated on application to coherence and relaxation in tunneling systems (see, eg., refs. 7, 17, 18, 19, 20, 21]), to decoherence in mesoscopic conductors 22, 23], superconducting qubits [7, 24], and nanomagnets 25]. It is clear that in the limit of weak coupling to the spin bath, it should be possible to map to an oscillator bath- studies in this limit appear in, eg., refs. [7, 19, 26, 27].

One can think of this breakdown of the oscillator bath model in several ways. One is dealing here with a breakdown of the assumption of weak system-bath couplings, and a corresponding breakdown of linear response in the behaviour of the bath dynamics (for more detailed discussion of this see ref. [], and refs. therein, and also the nice short summary by Weiss, in ref. [1], pp. 4952). We emphasize that the coupling to localised bath modes is almost always weak compared to the tunneling 
barrier energy $E_{B}$, or to the energy $\omega_{o}$ corresponding to small oscillation energies in the potential wells- so these modes are usually invisible in ordinary tunneling experiments. However, the energy scale of the localised bath modes, and their coupling to the central qubit coordinate, is often not small compared to the exponentially smaller tunneling energy $\Delta_{o}$. In the qubit regime it is the comparison with $\Delta_{o}$ that counts, particularly for decoherence (for a more precise discussion see below).

We are thus left with an interesting problem. What is the combined effect of spin bath and oscillator bath modes on the dynamics of the central system? In particular, how is decoherence affected by these two, and how does the oscillator bath take over from the spin bath as one goes to higher temperatures, or increases $\Delta_{o}$ ? Essentially one has to reconsider the whole question of the crossover between quantum and classical regimes when both baths are included.

In this paper we give a progress report on these questions for 2 kinds of crossover, viz:

(i) The crossover between spin bath controlled decoherence, which dominates when $\Delta_{o}$ is small, and the oscillator bath-controlled decoherence, which dominates when $\Delta_{o}$ is large (in both cases, assuming low temperature). The most interesting behaviour is in the crossover regime itself, when the decoherence goes to a minimum. Thus by raising $\Delta_{o}$ one can go from an incoherent regime, through a regime of coherent qubit dynamics, and then back to incoherent tunneling. To illustrate the idea we show how the general idea works for nanomagnets coupled to nuclear spins and phonons- The detailed application to specific magnetic and superconducting systems is discussed elsewhere 25, 28].

(ii) We look at how the spin bath influences the crossover between the quantum tunneling and classical thermally activated regime. This also involves a crossover between spin bath and oscillator bath environments. Given the complexity of this crossover, we do not attempt any complete discussion, but instead make some qualitative remarks on the physics, and then present some results for magnetic insulators (again involving phonons and nuclear spins). For related work one may go to a series of papers 29] on the application to ensembles of tunneling magnetic molecules.

\section{2: CROSSOVERS FROM COHERENCE TO INCOHERENCE}

The spin-boson model has a control parameter $\Delta_{o}$ (the operating frequency of the qubit); and we consider here the crossover between the small $\Delta_{o}$ regime, where decoherence is controlled by the spin bath, and the large $\Delta_{o}$ regime, where it is controlled by the oscillator bath. The interesting thing is that in the crossover between these 2 regimes lies a 'dead zone' where decoherence can be very low. This 'coherence window' will be very important for solid-state based quantum information processing.

\section{2a: QUBIT COUPLED TO OSCILLATOR AND SPIN BATHS}

We consider a 2-level system (a qubit) with the usual bare Hamiltonian

$$
\mathcal{H}_{o}=\Delta_{o} \hat{\tau}_{x}+\epsilon_{o} \hat{\tau}_{z}
$$

This is coupled to both spin and oscillator baths. The thermal energy $k_{B} T$, and the longitudinal and transverse field energies $\epsilon_{o}, \Delta_{o}$, are assumed to be much less than the energy gap $E_{g}$ to any higher levels of the system. In a magnetic qubit (eg., a magnetic molecule, or a rate earth ion), this 'spin gap' is typically $5-10 \mathrm{~K}$, and in a superconducting qubit the corresponding Josephson plasma frequency depends strongly on the junction geometry, and might be a little less.

The baths themselves are assumed to have Hamiltonians [1, 6, 7]:

$$
\begin{aligned}
\mathcal{H}_{o}^{o s c} & =\frac{1}{2} \sum_{q}\left[\frac{p_{q}^{2}}{m_{q}}+m_{q} \omega_{q}^{2} x_{q}^{2}\right] \\
\mathcal{H}_{o}^{S B} & =\omega_{k}^{\perp} \hat{m}_{k} \cdot \sigma_{k}+\sum_{k k^{\prime}} V_{k k^{\prime}}^{\alpha \beta} \sigma_{k}^{\alpha} \sigma_{k^{\prime}}^{\beta}
\end{aligned}
$$

in terms of a set of oscillators $\left\{x_{q}\right\}$ describing delocalised modes and a set of spins $\left\{\sigma_{k}\right\}$ describing localised modes (here for simplicity assumed to be a set of Pauli spin-1/2 systems). We have written the set of 'fields' $\left\{\mathbf{h}_{k}\right\}$, acting on the individual bath spins, in the form $\mathbf{h}_{k}=\omega_{k}^{\perp} \hat{m}_{k}$, where $\hat{m}_{k}$ is a unit vector in the direction of the field. We assume that a UV cutoff $\Omega_{0}$ exists in these Hamiltonians, so that all spin and oscillator degrees of freedom have energy $<\Omega_{o}$. The 2 baths are coupled to the central qubit via the following diagonal couplings:

$$
\mathcal{H}_{\text {int }}=\hat{\tau}_{z}\left[\sum_{q} c_{q}^{\|} x_{q}+\sum_{k} \omega_{k}^{\| \hat{l}_{k}} \cdot \hat{\sigma}_{k}\right]
$$

where $\left\{\hat{l}_{k}\right\}$ are a set of unit vectors. There can also be non-diagonal couplings, ie., terms which operate only when the qubit is switching between the eigenstates $|\uparrow\rangle$ and $|\downarrow\rangle$ of $\hat{\tau}_{z}$. These are usually specified by modifying the form of the transverse term $\Delta_{o} \hat{\tau}_{x}$ in the bare qubit Hamiltonian. For the oscillator bath one adds a coupling 30 ]

$$
\frac{1}{2}\left(\hat{\tau}_{+} c_{q}^{\perp} x_{q}+H . c .\right)
$$

and for the spin bath one makes the substitution

$$
\Delta_{o} \hat{\tau}_{x} \rightarrow \frac{1}{2}\left[\Delta_{o} \hat{\tau}_{+} e^{i \sum_{k} \alpha_{k} \mathbf{n}_{k} \cdot \vec{\sigma}_{k}}+H . c .\right]
$$


where the $\left\{\mathbf{n}_{k}\right\}$ are unit vectors.

We briefly note the important features of these interactions. First, we recall that the usual longitudinal spinboson couplings $\left\{c_{q}^{\|}\right\}$are typically $\sim O\left(N_{o}^{-1 / 2}\right)$, where $N_{o}$ is the number of oscillator degrees of freedom in the Hilbert space (defined by the UV cutoff $\Omega_{o}$ ). On the other hand the spin bath couplings $\left\{\omega_{k}^{\|}\right\}$may have a quite different dependence- in magnetic qubit systems they are usually independent of $N_{s}$, the number of spins, whereas in SQUID qubit systems one has $\omega_{k}^{\|} \sim O\left(N_{s}^{-2}\right)$, at least in the simplest designs. For large $N_{o}$ this means that the oscillator bath couplings are very weak- justifying the initial model of linear weak couplings. In the case of a SQUID qubit coupled to a spin bath one sees that it ought to be possible to map the problem to a spin-boson model, and indeed one can [7, 28]. However this is not an option for magnetic qubits- not only are the individual hyperfine couplings between the qubit and the nuclear spins independent of the number $N_{s}$ of nuclear spins, they are also large- in many cases $\omega_{k}^{\|}$for a single nuclear spin can exceed $\Delta_{o}$ ! In this case we must deal directly with the spin bath, and give up any hope of mapping the problem to a spin-boson model.

A second remark concerns the non-diagonal couplings. In cases where the diagonal couplings happen to be zero (which can happen under unusual circumstances) the non-diagonal couplings are the only remaining decoherence mechanism- this makes them very interesting for studies of decoherence (a point which has also been noted in recent discussions of superconducting qubits 31]). On the other hand when the diagonal couplings are nonzero, they usually dominate over the non-diagonal ones, at least when the qubit is modelling a tunneling solidstate system. It then follows that both $c_{q}^{\perp} / c_{q}^{\|}$and $\alpha_{k}$ are small- in fact $c_{q}^{\perp} / c_{q}^{\|} \sim O\left(\Delta_{o} / \Omega_{o}\right)$, and $\alpha_{k} \sim O\left(\omega_{k}^{\|} / \Omega_{o}\right)$ (for more details on this see refs. [7, 18]).

Finally, we note that the interactions between bath modes are treated differently in the oscillator and spin bath cases. In the oscillator bath case it is is usually argued that any weak anharmonic interactions have little relevance to the dissipation or decoherence caused by the bath- that information and energy are quickly transported away from the qubit, and so we can drop all reference to intra-bath interactions. In the case of the spin bath, however, it is clearly incorrect to drop such interactions- even though they are usually very small. This is because the spin bath describes local modes, which are not weakly coupled to the qubit- accordingly a large amount of energy and information can in principle be dumped into each mode and the $V_{k k^{\prime}}^{\alpha \beta} \sigma_{k}^{\alpha} \sigma_{k^{\prime}}^{\beta}$ interaction is the only way this can be redistributed. Over long time scales non-linear effects become inevitable, and the size of $V_{k k^{\prime}}^{\alpha \beta}$ becomes very important. We shall see how this works below.

\section{2b: DECOHERENCE RATES}

We define the decoherence dynamics for the qubit in a fairly standard way [1] by assuming an initial state $|\uparrow\rangle$, and calculating the reduced density matrix as a function of time thereafter, once the spin and oscillator baths are integrated out. The general form of the result (assuming the bias $\epsilon_{o}=0$ for simplicity) is

$$
\begin{aligned}
& 2 \rho(t)= \\
& \left(\begin{array}{cc}
\left(1+e^{-\Gamma_{1}(t)} \cos \left(2 \Delta_{o} t\right)\right) & i e^{-\Gamma_{2}(t)} \sin \left(2 \Delta_{o} t\right) \\
-i e^{-\Gamma_{2}(t)} \sin \left(2 \Delta_{o} t\right) & \left(1-e^{-\Gamma_{1}(t)} \cos \left(2 \Delta_{o} t\right)\right)
\end{array}\right)
\end{aligned}
$$

Here $\Gamma_{\mu}(t)$, with $\mu=1,2$, may have a complicated time dependence. If $\Gamma_{\mu}(t) \rightarrow \Gamma_{\mu}=$ const., (so that the coherence decays exponentially in time) one can write $1 / \Gamma_{\mu} \equiv T_{\mu}$, following NMR terminology. In this case we say that, in this basis, the decay rate $1 / T_{2}$ of the off-diagonal matrix elements is the decoherence rate- it characterizes the rate at which interference between $|\uparrow\rangle$ and $|\downarrow\rangle$ states is lost. In other cases one can usually derive a characteristic timescale $\tau_{\phi}$ for the loss of coherence, and this is called the decoherence time. One also defines a dimensionless measure of the decoherence rate, given by

$$
\gamma_{\phi}=1 / \tau_{\phi} \Delta_{o}
$$

or its inverse, the 'decoherence quality factor', often defined as $Q_{\phi}=\pi / \gamma_{\phi}=\pi \tau_{\phi} \Delta_{o}$. This Q-factor tells us roughly the number of coherent oscillations of the system before decoherence sets in.

Here we first quickly recall the known results for decoherence in this kind of problem. The dimensionless decay rate $\gamma_{\phi}$ has the following contributions:

(i) Oscillator bath contributions: The decoherence rates here depend on the Caldeira-Leggett spectral density [1]. For the cases we are interested in one has the following results:

(a) Phonon decoherence: this is relevant when we deal with spin-phonon coupling in magnetic insulators. Typically one considers a spin $\mathbf{S}$ (representing a molecular spin or other nanomagnet), which truncates at low temperature to a magnetic qubit (when $k_{B} T \ll \Omega_{o}$, where $\Omega_{o}$ is the spin gap to the higher electronic excitations). For decoherence the most important coupling to $\mathbf{S}$ is the nondiagonal coupling $\sim S \Omega_{o}|\mathbf{q}|$ to acoustic phonons having a Debye energy $\theta_{D}$ (for a simple derivation of this see ref. [18], and for a thorough discussion of spin-phonon couplings see [32, 48]). Standard spin-boson methods [1, 6], applied to this coupling, give a contribution $\gamma_{\phi}^{p h}$ to $\gamma_{\phi}$ of perturbative (ie., golden rule) form; when the applied 
bias is zero one gets [18]:

$$
\gamma_{\phi}^{p h}=\left[\left(S \Omega_{o} \Delta_{o}\right)^{2} / \theta_{D}^{4}\right] \operatorname{coth}\left(\Delta_{o} / k_{B} T\right)
$$

which is very weak at low energies (ie., for $\Delta_{o} \ll \theta_{D}$ ). Although no qubit behaviour has yet been seen in magnetic systems, there are extensive experimental results for the effect of phonons on the spin dynamics of magnetic molecules.

(b) Electronic decoherence: This comes in when we need to analyse decoherence in SQUID flux qubits 9, 10, 33, 34] or Cooper pair box charge qubits [8, 35, 36, 37]. For example, in flux qubits tunneling between flux states $\pm \phi_{m}$, and with charging energy $E_{c}$, one has a dimensionless coupling $\alpha=\left(16 \phi_{m}^{2} \omega_{0} / E_{c}\right) Q^{-1}$ between SQUID flux and electronic bath, parametrised in terms of the SQUID $Q$-factor. In this case of Ohmic dissipation the decoherence rate is [1]

$$
\gamma_{\phi}^{e}\left(\Delta_{o}\right)=\frac{\pi}{2} \alpha \operatorname{coth}\left(\Delta_{o} / k_{B} T\right)
$$

where again we assume the system is in resonance.

(ii) Spin bath contributions: We write the contributions to the spin bath-induced decoherence in terms of the couplings introduced in (4) and (6). It is useful to also introduce another quantity $E_{o}$ which quantifies the total effect on a single qubit level of the coupling to the bath spins- we have

$$
E_{o}^{2}=\sum_{k}\left(\omega_{k}^{\|}\right)^{2}
$$

so that $E_{o}$ is just the half-width of the Gaussian envelope of $2^{N_{s}}$ spin bath states associated with each qubit state. This formula is easily generalised to include 'higher spin' bath spins (see below). In section 3 we say more about the structure of these bath spin states inside this $2^{N_{s}}$-fold manifold.

There are 3 spin bath contributions to the decoherence 7, 18]:

(a) Noise decoherence: spin diffusion inside the spin bath causes the longitudinal bias acting on $\hat{\tau}_{z}$ to fluctuate over a range $\Gamma_{M}$ in energy bias space, causing phase noise. There will be a characteristic timescale $T_{2}$ associated with this noise (over longer timescales the bias fluctuates over a larger range- see section 3 for more details). If $\Delta_{o}^{3} \ll T_{2}^{-1} \Gamma_{M}^{2}$, the noisy bias fluctuates rapidly compared to the molecular tunneling dynamics, causing incoherent tunneling (this is the 'fast diffusion' limit for the spin bath [] ). This case is illustrated in Fig. 1, which shows the way the two qubit levels are affected in time by the fluctuating bias. In the opposite extreme of large $\Delta_{o}$ one has a much smaller noise contribution to $\gamma_{\phi}$ of

$$
\gamma_{\phi}^{N}=N_{e f f} / \pi \Delta_{o} T_{2}
$$

where $N_{\text {eff }}$ is the number of bath spins which are active (the exact number depends on the particular system- typically $N_{\text {eff }} \sim O(N)$, but the exact fraction $N_{\text {eff }} / N$ can vary widely from one system to another). This noise contribution $\gamma_{\phi}^{N} \ll 1$ (ie., it only weakly affects coherence). The analogue of Fig. 1 for this case would show very small fluctuations, which hardly affect the dynamics.

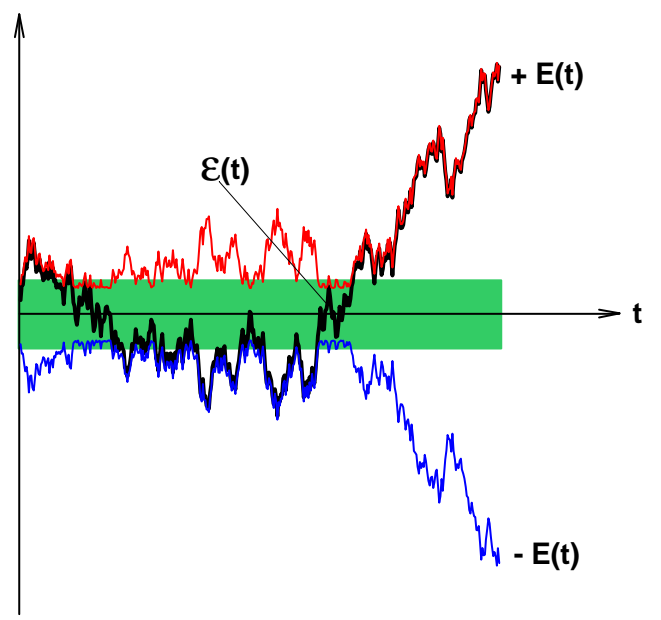

FIG. 1: We show the effect of a randomly fluctuating environmental noise bias $\varepsilon(t)$ (black curve) on a tunneling 2-level qubit with tunneling matrix element $\Delta_{o}$. The 2 levels having adiabatic energies $\pm E(t)$, with $E^{2}(t)=\Delta_{o}^{2}+\varepsilon^{2}(t)$, are shown as red $\&$ blue curves. The system can only make transitions when near "resonance" (ie., when $|\varepsilon(t)|$ is $\sim \Delta_{o}$ or less, the regions shown in green). The resulting dynamics of the qubit is incoherent in this case of strong noise.

(b) Precessional Decoherence: The field about which the $k$-th bath spin precesses changes each time the qubit flips, so that the time evolution of the spin bath states becomes entangled with that of the qubit. We can visualize this process by imagining the precessional motion of a bath spin in the qubit field (Fig. 2). Integrating out the spin bath then gives decoherence in the qubit dynamics. If the "operating frequency" $\Delta_{o}$ is low, ie., $\Delta_{o} \ll E_{o}$, then this "precessional decoherence" contribution $\gamma_{\phi}^{\kappa}$ to $\gamma_{\phi}$ is given by

$$
\begin{array}{rlrl}
\gamma_{\phi}^{\kappa} & =\frac{1}{2} \sum_{k}\left(\omega_{k}^{\|} / \omega_{k}^{\perp}\right)^{2} & & \left(\text { if } \omega_{k}^{\perp} \gg \omega_{k}^{\|}, \Delta_{o}\right) \\
\gamma_{\phi}^{\kappa}=\frac{1}{2} \sum_{k}\left(\omega_{k}^{\perp} / \omega_{k}^{\|}\right)^{2} & & \left(\text { if } \omega_{k}^{\|} \gg \omega_{k}^{\perp}, \Delta_{o}\right)
\end{array}
$$

One gets the second result from the first by a duality, switching the roles of $\omega_{k}^{\|}$and $\omega_{k}^{\perp}$ in the derivation of the first (cf. ref. [7], App. 2B).

If instead $\Delta_{o} \gg E_{o}$, ie., high operating frequency, then also $\Delta_{o} \gg \omega_{k}^{\|}, \omega_{k}^{\perp}$. The solution of this weak coupling problem is [7, 19]:

$$
\gamma_{\phi}^{\kappa}=\left(E_{o} / \Delta_{o}\right)^{2} / 2
$$

and this result is clearly important for the regime of coherent qubit dynamics. 
(c) Topological Decoherence: When the qubit flips, it causes a sudden time-dependent perturbation on the bath spins, described by the non-diagonal term (6). This induces transitions in the bath spin states, and a corresponding contribution to the entanglement of the bath spins and qubit states. Formally this entangles the topological Berry phase of the qubit [17] with that of the bath spins, in the same way as for precessional decoherence; after averaging over bath states the resulting contribution $\gamma_{\phi}^{\lambda}$ to $\gamma_{\phi}$ is

$$
\gamma_{\phi}^{\lambda}=\frac{1}{2} \sum_{k}\left|\vec{\alpha}_{k}\right|^{2}
$$

where $\left|\vec{\alpha}_{k}\right|=\pi\left|\omega_{k}^{\|}\right| / 2 \Omega_{0}$ is assumed to be small (for general coupling see refs. 7, 17]). In general this contribution is smaller than the precessional decoherence.

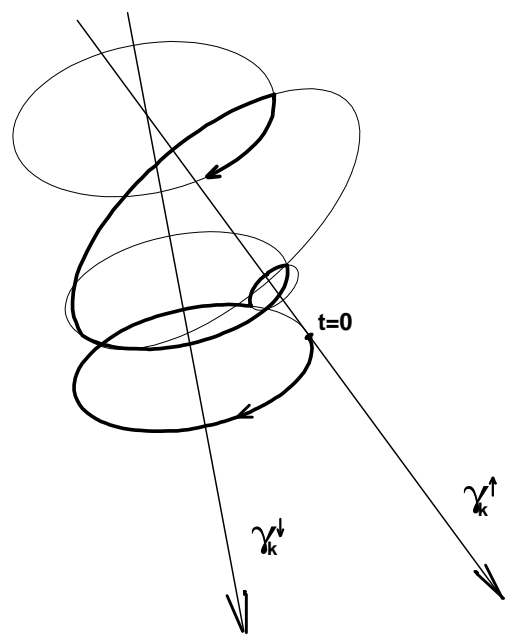

FIG. 2: We show schematically the motion of a satellite spin, in the presence of a qubit flipping between 2 different states $|\uparrow\rangle$ and $|\downarrow\rangle$. When the qubit flips, the qubit field acting on the $k$-th satellite spin rapidly changes, from $\gamma_{k}^{\uparrow}$ to $\gamma_{k}^{\downarrow}$ (or viceversa). Between flips the spin precesses around the qubit field, accumulating an extra "precessional" phase. Averaging over this phase gives precessional decoherence. The sudden change of qubit field also perturbs the satellite spin phase, giving further decoherence (the "topological decoherence" mechanism [17]).

We now observe, as has been noted before [38], that the spin bath decoherence rate is always higher at low energy (small $\Delta_{o}$ ), whereas the oscillator bath decoherence rate is higher at high energy (large $\Delta_{o}$ ). Thus there will be a 'coherence window' at intermediate values of $\Delta_{o}$, where $\gamma_{\phi}$ is small.

\section{2c: DECOHERENCE CROSSOVERS IN A MAGNETIC QUBIT}

At low $T$ the spin Hamiltonian of many large-spin nanomagnetic systems (magnetic molecules, rare earth ions, or nanomagnetic particles) reduces from that of a tunneling spin $\mathbf{S}$ to a simple 2-state form $\mathcal{H}_{o}(\hat{\tau})=$ $\left(\Delta_{o} \hat{\tau}_{x}+\epsilon_{o} \hat{\tau}_{z}\right)$, with the Pauli spin $\hat{\tau}$ acting on the 2 lowest spin levels [39, 40], as in our qubit Hamiltonian (10) . The spin gap $E_{g}$ to the next levels is typically $\sim 5-10 \mathrm{~K}$, and the 2 -state picture is valid at energies $\ll E_{g}$. We assume henceforth an "easy $\hat{z}$-axis" nanomagnet; then the 'bias' energy $\epsilon_{o}=g \mu_{B} S_{z} H_{o}^{z}$. When $\epsilon_{o}=0$, the splitting $\Delta_{o}$ between the 2 "qubit" states $|-\rangle,|+\rangle$ (bonding and anti-bonding eigenstates of $\left.\mathcal{H}_{o}(\hat{\tau})\right)$ is produced by tunneling between 2 potential wells, with each well having a "small oscillation" energy $\Omega_{o}$; typically $\Omega_{o} \sim E_{g}$. The qubit is thus the result of truncating out the higher spin states of the nanomagnetic system, which we should schematically in Fig. 3.

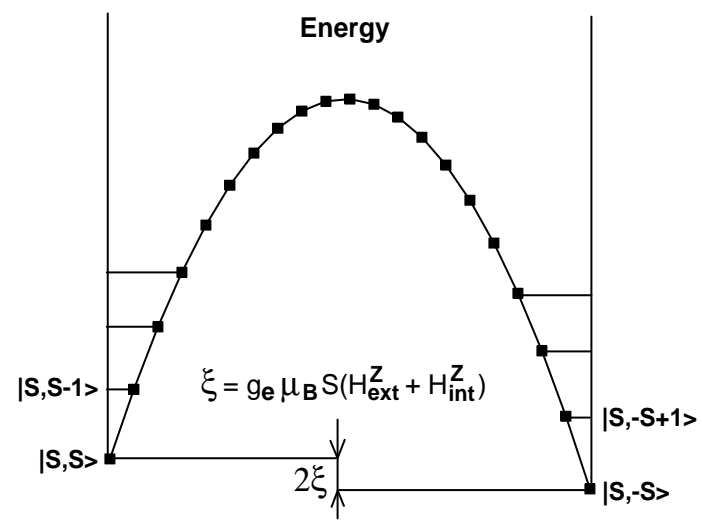

FIG. 3: Magnetic anisotropy barrier of a small magnetic system, such as a magnetic molecule. We show the eigenstates $|S, m\rangle$ of the longitudinal part $\mathcal{H}_{o}^{z}\left(S_{z}\right)$ of the spin Hamiltonian $\mathcal{H}_{o}(\mathbf{S})$. An external longitudinal field $H_{\text {ext }}^{z}$ (or an internal field $H_{i n t}^{z}$ ), biases the effective potential by an amount $\xi$. Adding transverse anisotropy terms to the spin Hamiltonian causes tunneling between the states in the figure. The 'ground state' tunneling amplitude $\Delta_{S}$ between states $|S, S\rangle$ and $|S,-S\rangle$ is called $\Delta_{o}$ in the text.

We define the states $|\uparrow\rangle,|\downarrow\rangle$ (eigenstates of $\hat{\tau}_{z}$ ) by $| \pm\rangle=|\uparrow\rangle \pm|\downarrow\rangle$. If the total nanomagnetic spin $\mathbf{S}$ is not too small, these states correspond roughly to semiclassical spin coherent states [1], having orientations $\mathbf{n}_{\sigma}$ (here $\sigma=\uparrow, \downarrow)$, which depend on both the internal anisotropy field of the nanomagnet, and any transverse external field $\mathbf{H}_{o}^{\perp}$. The splitting $\Delta_{o}$ depends sensitively on $\mathbf{H}_{o}^{\perp}$.

The intrinsic decoherence in insulating nanomagnets comes from entanglement of the nanomagnetic spin wave function with that of the nuclear spins and phonons [18]. We first see how to write these couplings in the form given in section 2(a). The details for the spin bath are a slight generalisation of this form, because the nuclear spins are not necessarily spin- $1 / 2$ objects.

The nuclear spins $\left\{\mathbf{I}_{k}\right\}$ couple to the electronic spins $\left\{\mathbf{s}_{j}\right\}$ in $\mathbf{S}$ (where $\mathbf{S}=\sum_{j} \mathbf{s}_{j}$ ) via hyperfine couplings

$$
\mathcal{H}_{\text {hyp }}=A_{\alpha \beta}^{j k} s_{j}^{\alpha} I_{k}^{\beta}
$$


whose form we do not specify here. We then define the field-dependent quantity

$$
\omega_{k}^{\|}=\frac{1}{2 I_{k}}\left|\sum_{j} A_{\alpha \beta}^{j k}\left(\left\langle s_{j}^{\alpha}\right\rangle^{\uparrow}-\left\langle s_{j}^{\alpha}\right\rangle^{\downarrow}\right) I_{k}^{\beta}\right|
$$

where $\left\langle s_{j}^{\alpha}\right\rangle^{\sigma}$ is the expectation value of $\mathbf{s}_{j}$ when $\mathbf{S} \rightarrow$ $S \mathbf{n}^{\sigma}$. The energy change of $\mathbf{I}_{k}$ when $\mathbf{S}$ flips from $S \mathbf{n}_{\uparrow}$ to $S \mathbf{n}_{\downarrow}$ is then $2 I_{k} \omega_{k}^{\|}$, ie., there is a diagonal coupling $\hat{\tau}_{z} \omega_{k}^{\|} \hat{l}_{k} \cdot \hat{\mathbf{I}}_{k}$ between the qubit and $\mathbf{I}_{k}$, where $\hat{l}_{k}$ is a unit vector parallel to the hyperfine field on $\mathbf{I}_{k}$. This is just the coupling specified in (4) in the last section.

The external transverse field $\mathbf{H}_{o}^{\perp}$ couples to $\mathbf{I}_{k}$ with Zeeman coupling $\omega_{k} \hat{m}_{k} \cdot \hat{\mathbf{I}}_{k}$, where $\omega_{k} \hat{m}_{k}=g_{k}^{N} \mu_{N} \mathbf{H}_{o}^{\perp}$ and $\hat{m}_{k}$ is a unit vector along $\mathbf{H}_{o}^{\perp}$. This is the same as the coupling given in (3). Finally, the interactions between the spins in the spin bath add a term

$$
\mathcal{H}_{N N}\left(\left\{\mathbf{I}_{k}\right\}\right)=\sum_{k=1}^{N} \sum_{k^{\prime}=1}^{N} V_{k k^{\prime}}^{\alpha \beta} \hat{I}_{k}^{\alpha} \hat{I}_{k^{\prime}}^{\beta}
$$

whose effect on the qubit will be handled by assuming that the spin diffusion caused by this weak interaction adds a "noise" term $\xi(t) \hat{\tau}_{z}$ to the static bias $\epsilon_{o} \hat{\tau}_{z}$. These terms taken together define an effective interaction Hamiltonian $\mathcal{H}_{N S}=\mathcal{H}_{o}(\hat{\tau})+V\left(\hat{\tau}, \mathbf{I}_{k}\right)$, where

$$
V=\hat{\tau}_{z}\left[\xi^{z}(t)+\sum_{k} \omega_{k}^{\|} \hat{l}_{k} \cdot \hat{\mathbf{I}}_{k}\right]+\sum_{k} \omega_{k} \hat{m}_{k} \cdot \hat{\mathbf{I}}_{k}
$$

To (19) we also add a 'spin-boson' coupling

$$
\mathcal{H}_{s \phi}=\sum_{q} c_{q}^{\perp} \hat{\tau}_{x} x_{q} \sim \sum_{q} S \Omega_{o}|\mathbf{q}| x_{q} \hat{\tau}_{x}
$$

to the acoustic phonon coordinate $x_{q}$. This non-diagonal term was already discussed above.

The energy scale over which the nuclear spin bath states operate is just the linewidth $E_{o}$ of the entire multiplet of nuclear states coupling to each qubit level [18]. It is clear that if the energy bias $\xi$ in the problem is less than $E_{o}$, it will be possible for the system to make tunneling transitions without the aid of the phonons, even if $\xi \gg \Delta_{o}$ (see Fig. 4). For this problem, with higher spin nuclei, one easily finds that

$$
E_{o}^{2}=\sum_{k} \frac{I_{k}+1}{3 I_{k}}\left(\omega_{k}^{\|} I_{k}\right)^{2}
$$

The acoustic phonon energy scale is the Debye energy $\theta_{D}$. Now in a nanomagnetic system the ratio $E_{o} / k_{B} \theta_{D}$ can be $\lesssim 10^{-4}$, suggesting the a very simple tactic for suppressing decoherence. If we tune $\Delta_{o}$ so that $k_{B} \theta_{D} \gg$ $\Delta_{o} \gg E_{o}$, then we will be in the "coherence window" mentioned above, where the qubit dynamics is too slow to disturb most phonons appreciably, but too fast for the nuclear spins to react.

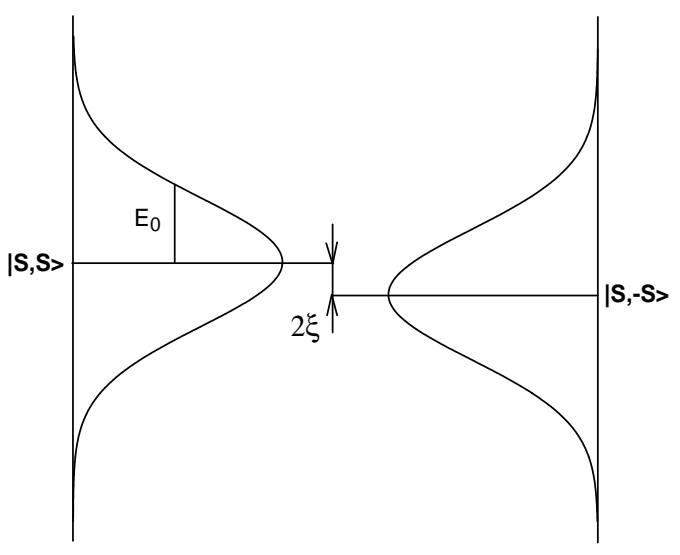

FIG. 4: The 2 qubit levels connected with the zero-field states $|S, S\rangle$ and $|S,-S\rangle$ each couple, via the internal hyperfine interactions, to a a very large number of nuclear spin levels. The result is a multiplet associated with each qubit level, which usually has a Gaussian density of levels, with a half-width $E_{o}$ (see text).

To substantiate this idea, we generalise the low field $\left(\Delta_{o}<E_{o}\right)$ calculations of nanomagnetic dynamics [18], where incoherent tunneling relaxation is found, to the high-field regime $\Delta_{o} \gg E_{o}$. Because the $\left\{\omega_{k}^{\|}\right\} \ll$ the Zeeman couplings $\left\{\omega_{k}^{\perp}\right\}$, and $\left\{\omega_{k}^{\|}\right\} \ll \Delta_{o}$, this dynamics can be solved [7, 19], by expanding (19) in $\omega_{k}^{\|} / \Delta$. If we first ignore the noise term, we get a new effective Hamiltonian

$$
\begin{aligned}
H_{N S} & =\left[\Delta_{o}+\sum_{k k^{\prime}} \frac{\omega_{k}^{\|} \omega_{k^{\prime}}^{\|}}{2 \Delta_{o}}\left(\hat{l}_{k} \cdot \mathbf{I}_{k}\right)\left(\hat{l}_{k^{\prime}} \cdot \mathbf{I}_{k^{\prime}}\right)\right] \hat{\tau}_{x} \\
& +\sum_{k} \omega_{k}^{\perp} \hat{m}_{k} \cdot \mathbf{I}_{k}+O\left(\left(\omega_{k}^{\|}\right)^{4} / \Delta_{o}^{3}\right)
\end{aligned}
$$

We can easily generalise the derivation of the result given in (14) for $\gamma_{\phi}^{\kappa}$ to the case where the nuclear spins have a spin modulus $I_{k}>1 / 2$. One finds easily that

$$
\begin{aligned}
\gamma_{\phi}^{\kappa} & =\sum_{k k^{\prime}} \sqrt{\frac{\left(I_{k}+1\right)\left(I_{k^{\prime}}+1\right)}{9 I_{k} I_{k^{\prime}}}} \frac{\omega_{k}^{\|} \omega_{k^{\prime}}^{\|} I_{k} I_{k^{\prime}}}{2 \Delta_{o}^{2}} \\
& =\frac{1}{2}\left(\frac{E_{o}}{\Delta_{o}}\right)^{2}
\end{aligned}
$$

Thus we see that the result (14) for precessional decoherence is generally valid, regardless of the size of the nuclear spins.

We now look at the 2 other contributions to the decoherence rate. The nuclear spin transitions induced directly by electronic spin flips add a contribution $\gamma_{\phi}^{\lambda}$ to $\gamma_{\phi}$. However when $\Delta_{o} \gg E_{o}$, the ratio $\gamma_{\phi}^{\lambda} / \gamma_{\phi}^{\kappa} \sim$ $O\left(\Delta_{o}^{2} / \Omega_{o}^{2}\right) \ll 1$, ie., the precessional decoherence always dominates over the topological decoherence.

The third contribution $\gamma_{\phi}^{N}$ comes from nuclear spin noise (the term $\xi(t) \hat{\tau}_{z}$ ). When $\Delta_{o} \gg E_{o}$, these fluctuations are extremely slow compared to $\Delta_{o}$; typically 
$T_{2} \sim$ msecs at low $T$, where $T_{2}$ is the typical transverse nuclear relaxation time for the $N$ nuclei controlling these fluctuations. One then gets $\gamma_{\phi}^{N}=N / \pi \Delta_{o} T_{2}$; we will see this is very small.

This summarizes the nuclear spin terms. The phonon contribution is as described above in (9), and in the low $T$ limit we are interested in, where $k_{B} T<\Delta_{o}$, we have:

$$
\gamma_{\phi}^{p h} \rightarrow\left[\left(S \Omega_{o} \Delta_{o}\right)^{2} / \Theta_{D}^{4}\right]
$$

At temperatures above $\Delta_{o}$ the phonon decoherence rate increases.

Now, since $\gamma_{\phi}^{\kappa}$ dominates nuclear spin decoherence, we can get an estimate for the optimal decoherence rate $\gamma_{\phi}^{\text {min }}$ by simply minimizing $\gamma_{\phi}^{\kappa}+\gamma_{\phi}^{p h}$ with respect to $\Delta_{o}$, assuming $k_{B} T<\Delta_{o}$, to get:

$$
\gamma_{\phi}^{\min } \approx \sqrt{2} S \Omega_{o} E_{o} / \theta_{D}^{2}
$$

at an optimal tunneling splitting $\Delta_{o}^{o p t}$ :

$$
\Delta_{o}^{(o p t)} \approx \theta_{D}\left(E_{o} / \sqrt{2} S \Omega_{o}\right)^{1 / 2} .
$$

We see that decoherence is optimised for a given $S$ by making $E_{o}$ and $\Omega_{o}$ small, and $\theta_{D}$ large, within the constraint that $\Omega_{o} \gg \Delta_{o}>k_{B} T$. If $k_{B} T>\Delta_{o}$ we get a different (less favorable) answer.

The detailed application of this kind of result to a nanomagnet, in cases where one knows something about the couplings, is in principle very useful for designing magnetic qubits. The tunneling splitting is most easily modified just by applying a magnetic field transverse to the easy axis- this can be used to tune $\Delta_{o}$ over many orders of magnitude (see Fig. 5). For more details of such applications, see ref. 25].

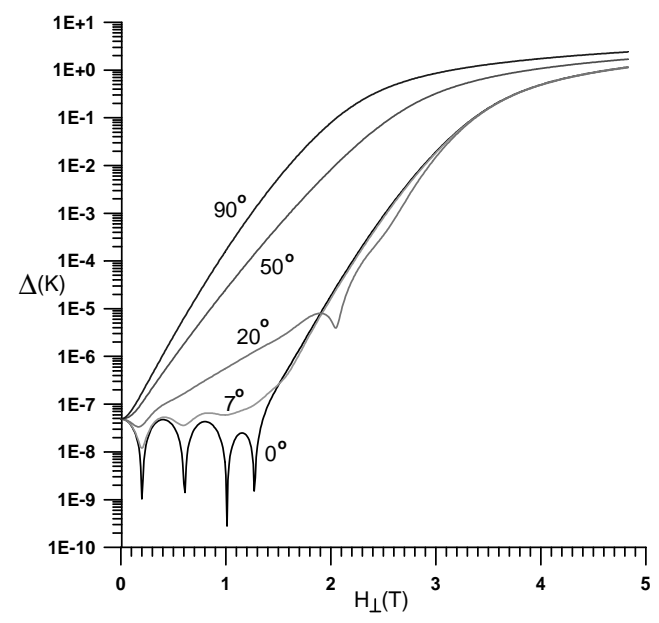

FIG. 5: The tunneling splitting $\left|\Delta_{o}\right|$ in the Fe-8 molecule, which is a good example of a tunneling nanomagnet. The tunneling anisotropy potential for the spin is biaxial, with an easy $z$-axis and a hard $x$-axis. The tunneling splitting is shown as a function of a transverse field $H_{\perp}$ oriented in the $x y$-plane, at an angle $\phi$ from the hard axis.

\section{3: INTERLUDE-SPIN BATH DYNAMICS}

In the results given above for decoherence rates, the intrinsic dynamics of the oscillator and spin baths played only a secondary role. In both cases it was assumed that phase information exchanged between system and bath was lost once it was taken up by the bath. This may not always be realistic, particularly in the spin bath- one can easily imagine situations in which the spin bath is cycled so as to recover some of this phase information, and in NMR this is actually done (eg., in 'multiple quantum coherence' experiments 42]). Even for oscillator baths it is well known that anharmonic oscillator couplings can allow the bath to hold information in certain modes for very long times- now a very well-studied phenomenon [43].

The question of the intrinsic bath dynamics is also important when one looks at the crossover between quantum and classical relaxation (next section). Therefore here we clarify what is and is not contained in the models we use.

As described by equation (3), the oscillator bath has the very simple dynamics of $N$ independent oscillators, with frequencies $\left\{\omega_{q}\right\}$. Coupling to the 'central system' hardly changes this dynamics, since the coupling strengths $c_{q} \sim O\left(N^{-1 / 2}\right)$. Thus nowhere in this model does energy and phase relaxation occurs in the bathboth simply accumulate independently in each mode. In reality anharmonic couplings cause rapid relaxation in systems of extended modes like electrons, phonons, magnons, etc; the only system to which (3) strictly applies is a bath of photons in a vacuum, in which the very weak vacuum polarisation-induced photon-photon interactions have been dropped. The reason that models like the spin-boson model work in most (but not all!) cases is just because this relaxation is usually fast- once energy or phase information has gone from the central system into a particular mode, it is rapidly diffused into other modes and so hard to recover.

In the case of the spin bath dynamics one has to be more careful. As discussed in detail in ref. 7], one can classify the large number of spin bath states into 'polarisation groups', defined by their total polarisation $M=\sum_{k} \hat{z}_{k} \cdot \sigma_{k}$ along some set of axes $\left\{\hat{z}_{k}\right\}$, where the $\left\{\hat{z}_{k}\right\}$ are unit vectors. If the 'external' field strengths $\left\{\omega_{k}^{\perp}\right\}$ are weak, so that $\omega_{k}^{\perp} \ll \omega_{k}^{\|}$, then it makes sense to have these axes along the direction of the local fields acting on the $\left\{\sigma_{k}\right\}$, coming from the qubit, ie., to make $\hat{z}_{k}=\hat{l}_{k}$ (compare eqtn (4)). If on the other hand the external fields dominate, one instead assumes that $\hat{z}_{k}=\hat{m}_{k}$, ie., the axes of quantization defining $M$ are just the external field directions. If these fields $\mathbf{h}_{k}=\omega_{k}^{\perp} \hat{m}_{k}$ are indeed defined by some strong external magnetic field $\mathbf{H}_{o}=\hat{n}_{o} H_{o}$, then $\hat{z}_{k}=\hat{n}_{o}$ for all spins in the bath, ie., the axis defining the bath polarisation is just the exter- 
nal field direction. However we emphasize that in many cases the fields $\mathbf{h}_{k}$ may have nothing to do with any external magnetic field. For example, the $\left\{\sigma_{k}\right\}$ might refer to a set of defects in a glass- in this case they couple to a strain field like electric dipoles. Even in the case of real spins the $\mathbf{h}_{k}$ may not be external fields but internal ones. For example, many tunneling nanomagnets do not tunnel between oppositely spin states, because the magnetic anisotropy field does not have uniaxial symmetry. In this case the hyperfine field on the nuclear spins does not flip through $180^{\circ}$ when the nanomagnet tunnels. One then resolves the hyperfine field into 2 components; $\mathbf{h}_{k}$ defines that component which does not change during the flip, while $\omega_{k}^{\|} \hat{l}_{k}$ defines a component which flips through $180^{\circ}$.

The point of defining polarisation groups in this way is that it then makes sense to define 2 relaxation times $\tilde{T}_{1}$ and $\tilde{T}_{2}$, such that $\tilde{T}_{1}$ defines the relaxation of $M$, and $\tilde{T}_{2}$ the spin bath relaxation within a given polarisation group. Note that even if the spin bath is actually made up of nuclear spins, these times are not the $T_{1}, T_{2}$ times measured in a typical NMR experiment, which only looks at a single nuclear species at a time- the times here refer to the whole spin bath. One can imagine an NMR experiment which polarizes a particular nuclear species, and then observes rapid relaxation of this polarisation into other nuclear species, via the inter-spain interactionseven though the total spin along the field is conserved (so that the spin bath $\tilde{T}_{1}$ defined here is still infinite).

Usually one expects $\tilde{T}_{1} \gg \tilde{T}_{2}$ at low $T$, because the interspin couplings $V_{k k^{\prime}}^{\alpha \beta} \sigma_{k}^{\alpha} \sigma_{k^{\prime}}^{\beta}$ can mediate transverse spin-spin relaxation processes, ie., cause spin diffusion in the spin bath and contribute to $\tilde{T}_{2}$, whereas $\tilde{T}_{1}$ processes typically require interaction with some external system. The most typical case where the bath spins inter-communicate by magnetic or electric dipolar interactions is actually hard to analyse theoretically, because these interactions are marginal in 3 dimensions (integrals of the form $\prod_{j}^{n-1} \int d^{3} \mathbf{r}_{j} / r_{j}^{3}$ appear in the calculation of the relaxation dynamics of $n$ coupled bath spins, so that multi-spin couplings are just as important as pairwise interactions, and distant spins as important as nearby ones 51]). Thus we would not usually try to calculate the spin bath $\tilde{T}_{2}$.

Let us now emphasize one of the crucial differences between the spin and oscillator baths. This is that because the $\left\{\omega_{k}^{\|}\right\}$are not weak, the spin bath dynamics depends very strongly on what the central system is doing. In the case of a central qubit one can imagine 2 extreme scenarios:

(i) We freeze the qubit dynamics by applying a longitudinal field $\epsilon_{o} \gg \Delta_{o}$. Then the spin bath dynamics is described by the effective Hamiltonian

$$
\mathcal{H}_{S B}=\sum_{k} \mathbf{b}_{k} \cdot \sigma_{k}+\sum_{k k^{\prime}} V_{k k^{\prime}}^{\alpha \beta} \sigma_{k}^{\alpha} \sigma_{k^{\prime}}^{\beta}
$$

where the static fields $\left\{\hat{\mathbf{b}}_{k}\right\}$ are given by

$$
\mathbf{b}_{k}=\omega_{k}^{\perp} \hat{m}_{k} \pm \omega_{k}^{\|} \hat{l}_{k}
$$

with the sign \pm depending on whether the central qubit is frozen in the $|\uparrow\rangle$ or $|\downarrow\rangle$ state.

We see that in this extreme case the bath would have its total polarisation conserved, provided we defined the polarisation groups using axes $\left\{\hat{z}_{k}\right\}$ parallel to the $\left\{\hat{\mathbf{b}}_{k}\right\}$. Actually we would not normally do this, but one can easily imagine a situation in which the applied field is either very strong or very weak, and then $M$ would be almost exactly conserved, ie., $\tilde{T}_{1}$ would be very long.

Now suppose we switch on the qubit dynamics- the easiest way to do this is to remove the bias field $\epsilon_{o}$. Then the nuclear bath finds itself subject to a quite different time-dependent Hamiltonian, of the form

$$
\begin{aligned}
\mathcal{H}_{S B}(t) & =\sum_{k} \omega_{k}^{\perp} \hat{m}_{k} \cdot \sigma_{k}+\sum_{k k^{\prime}} V_{k k^{\prime}}^{\alpha \beta} \sigma_{k}^{\alpha} \sigma_{k^{\prime}}^{\beta} \\
& +\tau_{z}(t) \sum_{k} \omega_{k}^{\|} \hat{l}_{k} \cdot \hat{\sigma}_{k}
\end{aligned}
$$

where the time-dependent variable $\tau_{z}(t)$ is jumping back and forth between \pm 1 with some correlation time $\sim$ $1 / \Delta_{o}$. This Hamiltonian described the spin bath now subject to an external 'telegraph noise', which causes transitions between different polarisation groups of the system- this will be true no matter how these polarisation groups are defined, provided both the $\left\{\omega_{k}^{\perp}\right\}$ and the $\left\{\omega_{k}^{\|}\right\}$ are non-zero. Suppose, for example, that we have a weak external field. Then $M$ is defined as $M=\sum_{k} \hat{m}_{k} \cdot \sigma_{k}$, as discussed above. We can imagine an initial state where all bath spins are oriented parallel or antiparallel to the initial local fields- but as soon as the qubit flips, they begin to precess. As discussed above, this is what causes precessional decoherence.

However, as observed already above, the motion of the central qubit depends itself on the spin bath dynamicswe must never forget that the telegraph noise acting on the spin bath depends in turn on the spin bath state. The simplifying feature is that energy conservation imposes a simple constraint on the allowed bath dynamics. In general the qubit will be off resonance by some energy $\xi$, the sum of an external field contribution and the internal field from the spin bath:

$$
\xi=\epsilon_{o}+\sum_{k} \omega_{k}^{\|} l_{k} \cdot \sigma_{k}
$$

In the simplest case where the couplings $\omega_{k}^{\|}$are either dominated by a single value $\omega_{o}^{\|}$, or else all cluster around this value (this happens in, eg., rare earth magnets like the $\operatorname{LiHo}_{x} Y_{1-x} F_{4}$ system [52], where the Ho hyperfine coupling to the $H o$ nuclear spin is much larger than its coupling to the other nuclear spins), we have approximately that $\xi \sim \epsilon_{o}+\omega_{o}^{\|} M$ (with some spread around the 
value $\omega_{0}^{\|}$, caused by dispersion in the values of the $\left.\omega_{k}^{\|}\right)$. Now for the qubit to make transitions we require that the initial and final energies $\pm \xi$ (for $\tau_{z}= \pm$ ) be the same within $\Delta_{o}$. This means that if $\epsilon_{o} \gg \Delta_{o}$, the central qubit can only flip if the spin bath absorbs the extra energy. However this will in general involve a change of $2 M$ in the net bath polarisation, such that $\omega_{o}^{\|}|M| \sim\left|\epsilon_{o}\right|$, each time the system flips. Thus at least $M$ bath spins have to flip- the time-varying field of the qubit must drive these transitions.

A formal calculation of the spin bath dynamics incorporates this constraint using a projection operator [], involving a dummy variable $\xi$ :

$$
\hat{\Pi}_{M}=\delta\left(\sum_{k=1}^{N} \hat{\sigma}_{k}^{z}-M\right)=\int_{0}^{2 \pi} \frac{d \xi}{2 \pi} e^{i \xi\left(\sum_{k=1}^{N} \hat{\sigma}_{k}^{z}-M\right)} .
$$

which restricts all bath states to the $M$-th polarisation group. Suppose now that the spin bath starts off with polarisation $M=M_{o}$, and that to maintain resonance, the net polarisation must change by $\pm 2 M$ each time the qubit flips from $|\uparrow\rangle$ to $|\downarrow\rangle$ or vice-versa, ie., it cycles between $M_{o} \longleftrightarrow M_{o}-2 M$. The simplest case arises if we ignore the interaction between bath spins completely, ie., let $V_{k k^{\prime}}^{\alpha \beta} \rightarrow 0$ in (29). Then one can write the dynamics of the bath in terms of operators $\hat{T}_{n}$ and $\hat{U}_{k}$, acting on the spin bath in the presence of $n$ flips of the qubit. The $\hat{T}_{n}$ are given by

$$
\begin{aligned}
\hat{T}_{n}= & {\left[e^{i \xi_{n} \sum_{k=1}^{N} \hat{\sigma}_{k}^{z}} \hat{U}^{\dagger} e^{i \xi_{n-1} \sum_{k=1}^{N} \hat{\sigma}_{k}^{z}} \hat{U}\right.} \\
& \left.\ldots \hat{U}^{\dagger} e^{i \xi_{1} \sum_{k=1}^{N} \hat{\sigma}_{k}^{z}} \hat{U}\right] .
\end{aligned}
$$

(involving a set of $n$ dummy variables) and the $\left\{\hat{U}_{k}\right\}$ define the change in the wave-function of the bath spins caused by the sudden flip of the qubit from one orientation to another, ie., the mismatch between in and out states:

$$
\left|\left\{\vec{\sigma}_{k}^{\text {out }}\right\}\right\rangle=\prod_{k=1}^{N} \hat{U}_{k}\left|\left\{\vec{\sigma}_{k}^{\text {in }}\right\}\right\rangle
$$

Suppose, eg., that $\omega_{k}^{\perp} \ll \omega_{k}^{\|}$, for all bath spins, ie., the field on each bath spin almost exactly reverses during each flip, through an angle $180^{\circ}-2 \beta_{k}$. Then $\hat{U}_{k}$ is just

$$
\hat{U}_{k}=e^{-i \beta_{k} \hat{\sigma}_{k}^{x}}
$$

Suppose we now want to write down the amplitude for the spin bath to start in the polarisation group $M_{o}$ and finish in the same polarisation group. This can only happen if the qubit flips $2 n$ times. The amplitude is then the sum of a term

$$
\begin{aligned}
G_{M_{o}, M}^{\uparrow \uparrow} & =\frac{\left(i \Delta_{o} t\right)^{2 n}}{(2 n) !} \prod_{i=1}^{2 n} \int \frac{d \xi_{i}}{2 \pi} e^{-i M_{o}\left(\xi_{2 n}+\xi_{2 n-1}+\ldots+\xi_{1}\right)} \\
& \times e^{2 i M\left(\xi_{2 n-1}+\xi_{2 n-3}+\ldots+\xi_{1}\right)} \hat{T}_{2 n}
\end{aligned}
$$

acting on the initial state of the spin bath, in which the qubit is assume to start and finish in the same state $|\uparrow\rangle$, and another terms in which it starts and finishes in the state $|\downarrow\rangle$.

The result of form (35) is only complete if energy conservation requires that the polarisation change by $\pm M$ each time. However in most cases there will be a wide range of values of $\omega_{k}^{\|}$, rather than a single dominant value, and so each polarisation group will be widely spread in energy space, and a large number of polarisation groups will have states at a given energy $\xi$ (ie., the groups will strongly overlap in energy space). In this more general case we should sum over transition amongst these groups with the appropriate weighting- the details are an obvious extension of what has just been described. In this way we can give a theoretical evaluation of the time $T_{1}$.

To calculate the full dynamics of the spin bath we must also include the action of the interspin interaction $V_{k k^{\prime}}^{\alpha \beta}$. This enables transitions amongst the different bath states inside the same polarisation group, even when $M$ does not change, ie., an evaluation of the spin bath $T_{2}$. We do not discuss here how such calculations may be done.

We underline here again the most important point of this interlude- that the spin bath controls the qubit dynamics, deciding whether the qubit may flip or not- but in its turn the qubit drives the spin bath dynamics.

\section{4: QUANTUM TO CLASSICAL CROSSOVER}

As noted in the introduction, a great deal is known about how the dynamics of a single quantum system, coupled to a thermal bath, changes as one raises the bath temperature 1, 3]. Many analyses use an oscillator model to describe the bath. However the models usually used to discuss this problem are restricted in certain ways. It is assumed that the bath stays in equilibrium during the times of interest, so that the internal relaxation times in the bath must be short compared to the timescale relevant to the dynamics of the quantum system, and energy given by the quantum system to the bath rapidly moves away, redistributing itself amongst bath modes.

The problem with such models is that at low $T$ the basic assumption of short internal relaxation times breaks down. This is of course well known and has been studied theoretically in, eg., spin glasses 44, 45], dipolar glasses [46], some models of low- $T$ phase nucleation [47], and in the various relaxation bottlenecks existing in magnetic systems [48] (to name only a few examples). However the problem is really generic to low- $T$ physics (a feature well-known to low- $T$ experimentalists [49], because it is the main obstacle to cooling to very low $T$ ). In fact, in almost all systems apart from pure liquid ${ }^{3} \mathrm{He}$ and ${ }^{4} \mathrm{He}$, the thermal bath has most of its low $T$ energy and entropy locked up in localised excitations. The relaxation of energy and entropy in and out of these modes ranges from 
$\mu$ secs to centuries. From a theoretical standpoint these facts are not surprising- they often arise in systems of local modes when couplings and fields are random (particularly when there is frustration of some kind in the interactions).

What this means here is that we should reconsider the whole problem of the quantum-classical crossover, using models which have these localised modes built in to them from the start. Consider, eg., the standard 2-well system, coupled now to both oscillator and spin baths. The oscillators represent delocalised modes, which at low $T$ are few in number but can move energy around quickly, and the spin bath represents the localised modes, which contain all the energy and entropy. A toy model for such a system is

$$
\begin{aligned}
\mathcal{H} & =\mathcal{H}_{o}(P, Q)+\mathcal{H}_{o}^{o s c}+\mathcal{H}_{o}^{S B}+V_{i n t} \\
\mathcal{H}_{o}(P, Q) & =P^{2} / 2 M+U_{o}(Q) \\
V_{\text {int }} & =Q\left[\sum_{q} c_{q} x_{q}+\sum_{k} \omega_{k}^{\|} l_{k} \cdot \hat{\sigma}_{k}\right]
\end{aligned}
$$

in which the oscillator and spin baths are as before (see eqtn. (3)), and the couplings are simple diagonal ones to the coordinate of the particle. One can have more complicated couplings, and in general one should also add counterterms [50] to $\mathcal{H}$ to renormalise the 2-well tunneling potential back to $U(Q)$.

Already in this toy model one begins to see how the quantum-classical crossover will work. At low $T$ the dynamics of the particle will be governed by its coupling to the spin bath, in the way previously described. Raising the bath temperature has little effect on the spin bath dynamics unless the $\left\{\omega_{k}^{\|}\right\}$are very large- they are already at high $T$ compared to their basic energy scales $\left\{\omega_{k}^{\|}, \omega_{k}^{\perp}\right\}$. However eventually the effect of thermal transitions of the oscillators to higher levels of the 2-well system begins to take effect. In the absence of the spin bath one sees a crossover to activated behaviour around a temperature $T_{o} \sim \omega_{o} / 2 \pi$, where $\omega_{o}$ is the (renormalised) small oscillation frequency of the system in one or other of the wells [1]. The width $\Delta T_{o}$ of the crossover depends on the details of the potential, the bath coupling, etc., but it will not be less than $\sim O\left(T_{o} / n\right)$, where $n$ is the number of levels below the barrier.

However the spin bath introduces new timescales in the problem, viz., the $\tilde{T}_{1}$ and $\tilde{T}_{2}$ introduced in the last section. Now these timescales, depending as they do on the dynamics of the central system itself, will decrease rapidly as we raise the temperature- the more rapid fluctuations of the central system, caused by coupling to thermally excited oscillators, stimulate more rapid transitions in the spin bath. This indirect effect of the oscillator bath on the spin bath dynamics, acting through the central system, is of course well known in NMR. In any case, we see the spin bath can compete with the oscillator bath over a rather wide range of temperatures in controlling the dynamics of the central system. This tells us that we may expect a much wider crossover between quantum and classical behaviour than occurs when one only deals with an oscillator bath environment (or only a spin bath environment).

Rather than give a general study of this crossover here, which is rather lengthy, we now present instead some relevant results for magnetic molecular spin relaxation, for which there also exist fairly detailed experimental results. These results actually capture some of the more general features of the problem.

\section{A: QUANTUM-CLASSICAL CROSSOVER FOR MAGNETIC MOLECULES}

It has been understood for many years that the thermally activated spin dynamics of insulating magnetic ions and nanomagnets (including large spin magnetic molecules) is driven by coupling to the phonon bath. Experimental investigations in the last 10 years of the tunneling relaxation dynamics of various magnetic molecules has led to many theoretical attempts to understand the temperature dependence of this behaviour in terms of the spin-phonon coupling to single tunneling spins 53, 54, 55, 56, 57]. Such calculations give a typical crossover from some straightforward low- $T$ tunneling to higher- $T$ activation- the details tend to be be messy because of the presence of many levels and different spinphonon coupling. These calculations must clearly apply at some sufficiently high temperature that neither nuclear spins nor intermolecular dipolar interactions are relevant. However they are not directly applicable to the experiments in the crossover regime, nor in the quantum regime. This is clear both on theoretical and experimental grounds, as follows:

From a theoretical point of view the interplay between nuclear and phonon couplings on a single nanomagnet cannot be ignored, especially given that the nuclear dynamics is fast. In experiments the intermolecular dipole interactions couple the relaxation of different molecules, so that they can only relax independently when $k_{B} T \gg V_{D}$, where $V_{D}$ is the strength of these dipolar interactions. Thus until we reach this rather high temperature (which in most experiments is well above the temperature $T_{o}$ defined earlier), both intermolecular dipole and hyperfine coupling to the nuclear spins must be included on an equal footing with the spin-phonon interactions.

From the experimental point of view the need for this is obvious. Even well above $T_{o}$ the relaxation is nonexponential in time (as in the quantum regime, but now with $T$-dependent characteristics), showing the molecules do not relax independently. Moreover, the 'hole-digging' phenomenon in the distribution of internal fields, caused by nuclear spins, also survives at temperatures well above 
$T_{o}$ (although with a time-development that becomes rapidly $T$-dependent), demonstrating that the nuclear spins are still partially controlling the tunneling dynamics.

To begin analysis of this problem we note that because the nuclear spin bath and the phonon bath do not interact with each other directly (but only via the central molecular spin), we can treat their relaxation rates as independent. We assume that each nanomagnet has a spin Hamiltonian of easy axis form $H_{o}(\mathbf{S})=$ $H_{o}^{z}\left(\hat{S}_{z}\right)+V_{o}^{\perp}\left(\hat{S}_{ \pm}\right)$, so that the transverse term $V_{o}^{\perp}$ causes tunneling between the eigenstates $|m\rangle$ of the longitudinal part (defined by $H_{o}^{z}|m\rangle=E_{m}^{(0)}|m\rangle$ ). Let us also assume that the applied longitudinal field is small, so that levels $|m\rangle$ and $|-m\rangle$ are near resonance (and level $|m\rangle$ is not near resonance with any other levels). Now suppose we start with an ensemble of such nanomagnets, all of them having $m=S$, ie., so the system is completely polarised. If we ignore any coherence effects (ie., assume incoherent relaxation), and also ignore intermolecular interactions, then the kinetic equation for the system reduces to

$$
\begin{aligned}
\dot{P}_{m}^{(1)}(\xi, \mathbf{r}, t)= & -\sum_{m^{\prime}}\left[\Gamma_{m^{\prime} m}(\xi, T) P_{m}^{(1)}(\xi, \mathbf{r}, t)\right. \\
& \left.-\Gamma_{m m^{\prime}}(\xi, T) P_{m^{\prime}}^{(1)}(\xi, \mathbf{r}, t)\right]
\end{aligned}
$$

$\left(\Gamma_{-m, m}=\Gamma_{m,-m} \equiv \Gamma_{m}\right)$ where $P_{m}^{(1)}(\xi, \mathbf{r}, t)$ is the 1molecule probability distribution, describing the probability to find a molecule at position $\mathbf{r}$, in state $|m\rangle$, in a bias field $\xi$, at time $t$; and $\Gamma_{m m^{\prime}}(\xi, T)$ is the rate at which nanomagnets in a local field $\xi$ make transitions from state $\left|m^{\prime}\right\rangle$ to $|m\rangle$, under the influence of both phonons (at a temperature $T$ ) and nuclear spins. The assumption of non-interacting phonon and nuclear baths implies we can write:

$$
\Gamma_{m m^{\prime}}(\xi, T)=\Gamma_{m m^{\prime}}^{N}(\xi, T)+\Gamma_{m m^{\prime}}^{\phi}(\xi, T)
$$

with individually defined nuclear spin- and phononmediated relaxation rates. The system can move up or down levels on the same side of the barrier by emission of phonons. Of particular interest here are the inelastic tunneling rates out of level $|m\rangle$ in a bias field, to the other side of the barrier. The phonon-mediated process of this kind has the form [58]:

$$
\Gamma_{m}^{\phi}(\xi) \approx \frac{\Delta_{m}^{2} W_{m}(T)}{\Delta_{m}^{2}+\xi_{m}^{2}+\hbar^{2} W_{m}^{2}(T)}
$$

where $W_{m}(T)$ is that part of the linewidth of the $m$ th level caused by phonon-mediated intra-well processes, $\xi_{m}=g \mu_{B} m H^{z}$ is the bias energy between levels $| \pm m\rangle$ (with the field $H_{z}$ the sum of internal and external fields), and $\Delta_{m}$ is the tunneling matrix element for tunneling between these same levels.
On the other hand for the nuclear spin bath-mediated rate we will use here the temperature-independent form:

$$
\Gamma_{m}^{N}(\xi) \sim \frac{2 \Delta_{m}^{2} G_{N}^{(m)}}{\pi^{1 / 2} \hbar E_{o}^{(m)}} e^{-\left|\xi_{m}\right| / E_{o}^{(m)}}
$$

where $E_{o}^{(m)}$ is a generalisation of the quantity $E_{o}$ which plays a role in nuclear spin-mediated tunneling in the quantum regime- roughly one has $E_{o}^{(m)} \sim(m / S) E_{o}$ (cf., eqtn. (11)). This quantity measures the range over which the nuclear spin bias is being swept, either by internal spin diffusion or by transitions caused by the nanomagnetic dynamics (the mechanism described in the last section). The factor $G_{N}^{(m)}=\exp \left\{-\Delta_{m}^{2} / 2\left(E_{o}^{(m)}\right)^{2}\right\}$ follows from the Gaussian spread of the nuclear multiplet, and simply says that $\Gamma_{m}^{N}(\xi)$ vanishes when $\Delta_{m}$ becomes large in comparison with $E_{o}^{(m)}$.

The rationale for using (40) is that we are interested here in time scales long compared to the $\tilde{T}_{1}$ and $\tilde{T}_{2}$ discussed in the last section. In this case we expect that the system is able to cover the whole range of states in the nuclear spin manifold surrounding each level, and so we can simply weight these according to their number, ie., according to a density of states. Clearly this approximation breaks down if we are interested in shorter time scales- we will not consider this problem here. We can in fact go further- since at time scales longer than all relaxation times, the nuclear bias will fluctuate across the whole range of nuclear states, of width $E_{0}^{(m)}$, we can incorporate this into the phonon rate as an average- for example, when $E_{0}^{(m)}>>\max \left\{\Delta_{m}, \hbar W_{m}\right\}$, the phonon rate becomes

$$
\Gamma_{m}^{\phi}(\xi, T) \approx \frac{\Delta_{m}^{2} W_{m}(T)}{E_{0}^{(m)} \sqrt{\Delta_{m}^{2}+\hbar^{2} W_{m}^{2}(T)}} \sqrt{\frac{\pi}{2}} e^{-\xi_{m}^{2} / 2\left(E_{0}^{(m)}\right)^{2}}
$$

Actually this result turns out to be valid even when the spin bath dynamics is slow compared to the timescale we are interested in- but with one simple modification. The point is that even if a given molecule in a dipolar bias field $\xi_{D}$, coming from the other molecules, cannot find resonance, because the spin bath brings it too slowly to resonance, nevertheless some fraction $x$ of the molecules in a field $\xi$ will find themselves in a compensating nuclear bias field near $-\xi_{D}$, near enough so that the molecule is quickly brought to resonance.

We see that in this very simple approximation the spin and oscillator bath-mediated relaxation processes already influence each other strongly, albeit in a rather trivial way. Because (39) reduces the contribution of the higher levels to the relaxation, which in the usual theory of the crossover take over very quickly from the lowest levels as one goes through $T=T_{o}$, the net effect is to broaden the crossover.

To illustrate this it is useful to show results for a particular system. We again choose the $F e-8$ molecule, al- 
ready discussed above in the context of low- $T$ decoherence. In Fig. 6 we show the contributions from the different levels to the relaxation as a function of $T$ for the $\mathrm{Fe}$ 8 system. To give results comparable with experiment we have generalised the kinetic equation (37) to include dipolar interactions- this development is a rather messy but fairly straightforward adaptation of the method used in ref. [59]. The main effect of adding these interactions is however not to change the width of the crossover, but rather to change the time dependence of the relaxationthis it is fairly complex, even in the quantum regime [59], and not relevant to the present study.

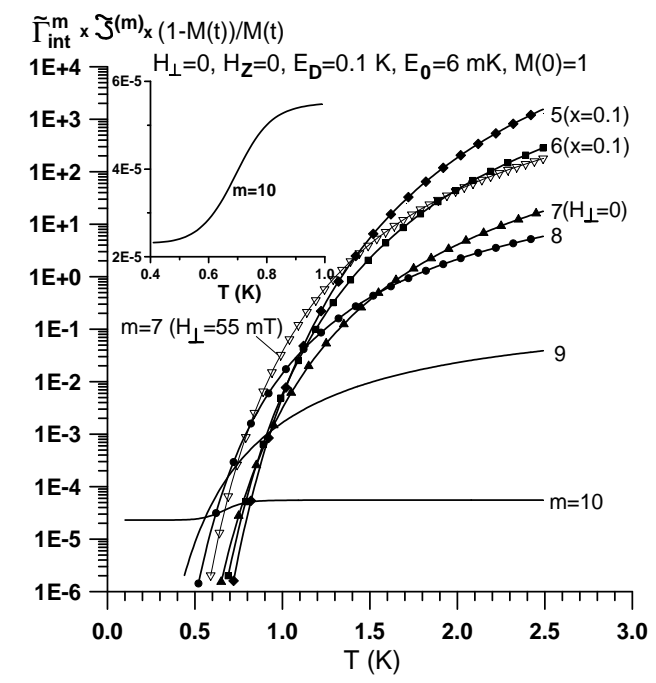

FIG. 6: The relaxation rates as a function of temperature for relevant values of $S_{z}=m$ in the case of the $F e_{8}$ system. The two curves for $m=7$ correspond to different transverse fields, viz., (i) $H_{\perp}=0$ (filled triangles); and (ii) $H_{\perp}=55 \mathrm{mT}$ (open flipped triangles). The inset enlarges the $m=10$ curve around $T \gtrsim T_{o}$, where $T_{o}$ is the temperature at which phonon transitions would normally cause the tunneling dynamics to rapidly crossover to activated behaviour.

The most important point to be noted from Fig. 6 is that the crossover is now very wide- it extends from $T \sim 0.4 K$ up to $T \sim 1.7 \mathrm{~K}$, above which temperature the contributions from level $m=5$ begin to dominate. It will be surprising to those familiar with the standard theory of the quantum-classical crossover that it is an intermediate level that dominates. The crossover is so wide for 2 reasons, viz.,

(i) The reason noted above, ie., the spreading of the levels by the nuclear spin hyperfine coupling (and in the case of this calculation, also the dipolar fields, so that now the spread is even greater than $\left.E_{o}^{(m)}\right)$. This emphasizes the role of the lower levels, more than would otherwise be the case; and

(ii) We see already that in the basic phonon transition rate in (39) there is a saturation in the rate for the high levels (having small $m$ and large $\Delta_{m}$ ). This is because the typical bias $\xi_{m}$ in this formula, and in the more general formula (41), will now be either a dipolar or nuclear hyperfine bias, which is much larger than the phonon linewidth $W_{m}$. Once $\Delta_{m}$ exceeds this bias, the presence of $\Delta_{m}$ in the denominator stops the rapid increase of the rate- this happens before one reaches the very highest levels, and is the basic reason why intermediate levels dominate the relaxation over a wide temperature range. In the case of $\mathrm{Fe}-8$, this happens for $m=5$, but clearly could happen for some intermediate level in a different system. In most of the earlier papers on phonon-mediated tunneling relaxation of nanomagnets, the factor of $\Delta_{m}^{2}$ in the denominator was not included- this led to a quite different picture of the crossover.

Actually experiments in these systems do show a very wide crossover. The detailed comparison between theory and experiment is rather interesting, since one may analyse both the relaxation as a function of time (and how the form changes with $T$, along with rate) and also the $T$-dependence of the hole-digging dynamics [29].

\section{SUMMARY}

This paper has not attempted a complete study- instead we have tried to make some general points about 2 kinds of crossover, uncluttered by too much detail for particular systems. To illustrate the general remarks it has been nevertheless useful to give results for the $\mathrm{Fe}-8$ molecule. It goes without saying that the detailed calculations for this system (and others, such as SQUIDs) are rather lengthy, and including them would have obscured the points we wishes to make.

The 2 main points are that

(i) The presence of the spin bath is very bad for decoherence when the basic energy scale $\Delta_{o}$ of a qubit is small. This means that the spin-boson model is not applicable at all in this regime- one must use a 'central spin' model [7]. However if we increase $\Delta_{o}$, the spin bath decoherence effects fall off extremely rapidly, and eventually become negligible. At this point the spin-boson model becomes applicable, and decoherence begins to increase again as one further increases $\Delta_{o}$, a well-known feature of the model [1].

(ii) In the standard quantum-classical crossover that occurs as one raises the bath temperature for a tunneling system, the presence of the spin bath is again very important. The main effect it has is first to completely change behaviour in the quantum regime, and then to enormously broaden the usual rather sharp crossover that exists when one only deals with an oscillator bath.

It is quite clear that these results are only the beginning of a proper study of the way in which spin and oscillator baths work together. The remarkable edifice of theoretical work that has been constructed around the spin-boson and related models [1] should be a very nice model for what interesting paths remain to be explored in this area. 
We thank J. Angles d'Auriac and J. de Jongh for hospitality while this article was being written, and the CIAR and NSERC in Canada, and grant number NS1767.2003.2 in Russia, for support.

[1] U. Weiss, 'Quantum Dissipative Systems' (World Scientific, 2nd edition, 1999)

[2] H.A. Kramers, Physica 7, 284 (1940)

[3] P. Hanggi, P. Talkner, M. Borkovec, Rev. Mod. Phys. 62, 251 (1990)

[4] "Quantum Tunneling in Condensed Matter", ed. Yu. Kagan, A.J. Leggett (Elsevier, 1992).

[5] U. Eckern, A. Schmid, Chapter 3 in Kagan and Leggett [4]

[6] A.J. Leggett et al., Rev. Mod. Phys. 59, 1 (1987).

[7] N.V. Prokof'ev, P.C.E. Stamp. Rep. Prog. Phys. 63, 669 (2000)

[8] Y. Nakamura, Yu. A. Pashkin, T. Yamamoto, J.S. Tsai, Phys. Rev. Lett. 88, 047901 (2002)

[9] C.H. Van der Wal, et al., Science 290, 773 (2000)

[10] C. van der Wal, Ph.D thesis (Delft, Sept. 2001)

[11] W. Wernsdorfer, A. Caneschi, R. Sessoli, D. Gatteschi, A. Cornia, V. Villar, C. Paulsen, Phys. Rev. Lett. 84, 2965 (2000)

[12] A. Morello et al., cond-mat/0211209 (2002), and to be published.

[13] Y. R. Shen, Phys. Rev. 155, 201 (1967)

[14] A.S. Davydov, A.R. Sherikov, Phys. Stat. Sol. b 51, 57 (1972)

[15] P.C.E. Stamp, Phys. Rev. Lett. 61, 2905 (1988)

[16] E. Shimshoni, Y. Gefen, Ann. Phys. 210, 16 (1991)

[17] N.V. Prokof'ev and P.C.E. Stamp, J. Phys. CM Lett. 5, L663-L670 (1993)

[18] N.V. Prokof'ev, P.C.E. Stamp, J. Low Temp. Phys., 104, 143 (1996).

[19] A.O. Caldeira, A.H. Castro-Neto, T. Oliveira de Carvalho, Phys. Rev. B48, 13974 (1993)

[20] N. Abarenkova, J.C. Angles d'Auriac, Phys. Lett. A219, 335 (1996)

[21] G. Levine, Phys. Rev. B56, 7815 (1997)

[22] Y. Imry, H Fukuyama, P. Schwab, Europhys. Lett. 47, 608 (1999)

[23] A. Zawadowski, J. von Delft, D.C. Ralph, Phys. Rev. Lett. 83, 2632 (1999)

[24] L. Tian, et al., pp. 429-438 in "Quantum Mesoscopic Phenomena and Mesoscopic Devices in Microelectronics", ed. I.O.Kulik, R. Ellialtioglu (Kluwer, 2000).

[25] P.C.E Stamp and I.S. Tupitsyn, cond-mat/0302015

[26] J. Shao and P. Hanggi, Phys. Rev. Lett. 81, 5710 (1998)

[27] K.M. Forsythe and N. Makri, Phys. Rev. B60, 972 (1999)

[28] P.C.E. Stamp, to be published

[29] I. Tupitsyn, P.C.E. Stamp, to be published

[30] Yu. Kagan, N.V. Prokof'ev, Chapter 2 in Kagan and Leggett [4]
[31] L.B. Ioffe et al., Nature 398, 679 (1999).

[32] P.Politi, A. Rettori, F. Hartmann-Boutron, J. Villain, Phys. Rev. Lett. 75, 537 (1995).

[33] J.E. Mooij et al., Science 285, 1036 (1999).

[34] I. Chiorescu, Y. Nakamura, C.J.P.M. Harmans, J.E. Mooij, Science 299, 1869 (2003)

[35] Y. Nakamura, Yu. A. Pashkin, J.S. Tsai, Nature 398, 786 (1999)

[36] D. Vion, A. Aasime, A. Cottet, P. Joyez, H. Pothier, C. Urbina, D. Esteve. M. Devoret, Science 296, 886 (2002)

[37] Yu. A. Pashkin, T. Yamamoto, O. Astafiev, Y. Nakamura, D. Averin, J.S. Tsai, Nature 421, 823 (2003)

[38] M. Dubé, P.C.E. Stamp, Chem. Phys. 268, 257 (2001)

[39] W. Wernsdorfer, Adv. in Chem. Phys. 118 (2001); /condmat 1010104.

[40] I.S. Tupitsyn, B. Barbara, pp. 109-168 in "Magnetoscience - from Molecules to Materials, vol. 3", ed. Miller \& Drillon (Wiley, 2001).

[41] A. Auerbach, "Interacting Electrons and Quantum Magnetism" (Springer-Verlag, 1994)

[42] C.P. Slichter, 'The Principles of Nuclear Magnetic Resonance', Springer, 1992.

[43] E. Fermi, J. Pasta, S. Ulam, Los Alamos report LA1940 (1955)[reprinted in E. Fermi, Collected papers II, p. 978 (Univ. Chicago press, 1965)]; J. Ford, Phys. Rep. 213, 271 (1992).

[44] K.H. Fischer, J.A. Hertz, 'Spin Glasses' (CUP, 1991)

[45] M. Mezard, G. Parisi, M.A. Virasoro, 'Spin Glass Theory and Beyond', World Scientific, 1987.

[46] A.L. Burin, J. Low Temp. Phys. 100, 309 (1995)

[47] See, eg., A.J. Leggett, S.K. Yip, ch. 8, p. 523 in 'Helium Three', ed. W.P. Halperin, L.P. Pitaevski (Elsevier, 1990); and refs. therein.

[48] A. Abragam, B. Bleaney, 'Electron Paramagnetic Resonance of Transition Ions', Clarendon Press (1970).

[49] F Pobell, 'Matter and Methods at Low Temperatures', Springer, 1995.

[50] A.O. Caldeira, A.J. Leggett, Ann. Phys. 149, 374 (1983)

[51] L. Levitov, Phys. Rev. Lett. 64, 547 (1990)

[52] W. Wu, B. Ellmann, T.F. Rosenbaum, G. Aeppli, D.H. Reich, Phys. Rev. Lett. 67, 2076 (1991); S. Ghosh, R. Parthsarathy, T.F. Rosenbaum, G. Aeppli, Science 296, 2195 (2002); R. Giraud et al., Phys. Rev. Lett. 87, 57203 (2001).

[53] J. Villain, F. Hatmann-Boutron, R. Sessoli, A. Rettori, Europhys. Lett. 27, 159, 1994

[54] D. Garanin, E.M. Chudnovsky, Phys. Rev. B56, 11102 (1997)

[55] F. Luis et al., Phys. Rev. B57, 505 (1998); J.F. Fernandez et al., Phys. Rev. Lett. 80, 5659 (1998)

[56] A. Fort et al., Phys. Rev. Lett. 80, 612 (1998)

[57] M.N. Leuenberger, D. Loss, Phys. Rev. B61, 1286 (2000)

[58] Yu. Kagan, L.A. Maksimov, Sov. Phys. JETP 52, 688 (1980).

[59] N.V. Prokof'ev, P.C.E. Stamp, Phys. Rev. Lett. 80, 5794 (1998); N.V. Prokof'ev, P.C.E. Stamp, J. Low Temp. Physics 113, 1147 (1998) 